\title{
Performance of different threshold estimation methods on SEMG wavelet de-noising in prolonged fatigue identification
}

\begin{abstract}
Surface electromyography (SEMG) signals are widely used in fatigue identification. Fatigue after high intensity exercise and sports training needs to be balanced with rest to allow biochemical reactions during sports activity to return to a normal level. Inadequate rest leads to prolonged fatigue $(\mathrm{PF})$ conditions such as musculoskeletal disorder, unexplained lethargy and performance decrement. Continuous sports training under these conditions may lead to injury. Fatigue identification at this stage is crucial since changes in amplitude and frequency of SEMG may determine whether the player is under normal fatigue (NF) or PF condition. During data collection, there are many interferences and noises which can reduce signal to noise ratio (SNR) of SEMG and affect PF detection. This paper pre-processed SEMG signals using Stationary Wavelet Transform (SWT) 'db' 45 with different threshold (Th) estimation techniques of de-noising such as RigRSURE, HeurSURE, minimax, universal threshold and a new estimation of threshold method which is based on a baseline of SEMG decomposition details. Naïve Bayes classification results using time and frequency features indicate that the new estimation of threshold method have the highest accuracy (98\%), compared to RigRSURE (85\%), HuerSURE (68\%), Universal Threshold (74\%) and minimax (76\%).
\end{abstract}

Keyword: Surface electromyography; Prolonged fatigue; Overtraining; Baseline noise; Wavelet thresholding; Stationary wavelet transform 